\title{
Fast Edge Detection Architecture using Different Levels of Parallelism on a FPGA
}

\author{
Mohammad Shokrolah Shirazi \\ Electrical \& Computer Engineering Department \\ University of Nevada, Las Vegas \\ 4505 South Maryland Parkway \\ Las Vegas, NV, 89154
}

\author{
Brendan Tran Morris \\ Electrical \& Computer Engineering Department \\ University of Nevada, Las Vegas \\ 4505 South Maryland Parkway \\ Las Vegas, NV, 89154
}

\begin{abstract}
Implementing edge detection techniques on a FPGA has recently become more popular since it benefits high speed which is desired for real-time applications. This work presents a fast FPGAbased architecture for first order derivative edge detection methods. Fast pipeline-based architectures are presented which are able to perform edge detection using different levels of parallelism to accelerate the process. This acceleration includes applying parallelism over convolution masks, edge detection modules and image intensity values. Two different edge detection architectures are proposed called one-way and two-way parallel methods. The architectures are implemented using Verilog HDL for a typical image and we synthesized them for Cyclone IV FPGA. Experimental results show the speed-up near to 460 and 920 for one-way and two-way parallel architectures.
\end{abstract}

\section{General Terms:}

Architecture, Real time applications, Speed-up

\section{Keywords:}

Edge detection, Parallelism, FPGA

\section{INTRODUCTION}

Nowadays, there is a new trend towards implementing image processing algorithms over a hardware rather than a software since it can more efficiently execute the algorithms [8]. As result, instead of general purpose processors that use software to run image processing algorithms, it is preferred to design a dedicated hardware for real-time image processing applications.

Some hardware approaches use Field Programmable Gate Array (FPGA) technology in their studies [2 4 8]. FPGA typically consists of a logic blocks' matrices such as look up tables, gates, flipflops and memories that are connected via programmable interconnections. The FPGA configuration is generally specified using Hardware Description Languages (HDL) similar to ApplicationSpecific Integrated Circuits (ASICs) which is custom manufactured for specific design tasks. FPGAs can be reprogrammed to form a desired application or functionality requirements after manufacturing. This feature distinguishes FPGAs from ASICs.
There are three major reasons that encourage researchers to implement their algorithms on the FPGA. First, all logics inside FPGA can be rewired, or reconfigured with a different design according to the designers' demand [14]. Second, the reconfigurable nature of FPGA allows us to implement different algorithms multiple times providing more flexibility in comparison with ASICs. Finally, the hardware nature of FPGA provides greater speed in comparison with software-based approaches since it uses different hardware modules that could be run in a parallel manner [3].

Edge detection is one of the popular image processing techniques that could be implemented on the FPGA. Image edges contain important information for creating popular image features which contain significant structural properties used in computer vision for object recognition, tracking and motion detection [17].

Edge detection is the process of locating brightness discontinuities between pixels in a digital image. The basic edge detection methods calculate the first order derivative by using some gradient operators such as Prewit [6, 10 11], Sobel [1 6, 11 |12 |15||16| 18] or Robert $|\overline{6}|$. In these methods, image gradients are obtained by introducing different kernel masks for performing convolution over the image. These masks are responsible for obtaining image gradients in two different directions. There are also some second order derivative methods such as Laplacian [17], Difference of Gaussian (DOG) [9] and Laplacian of Gaussian [7]. These methods find zero crossing of the second derivative to determine edges. However, second derivative methods are not usually used for edge detection since they are too sensitive to noise [15].

In this paper, we present a fast FPGA-based architecture for first derivative edge detection by performing either Sobel or (and) Prewit or (and) Robert operation on a given image. Each Sobel, Prewit or Sobel hardware modules benefit the pipeline structure running in parallel. The proposed edge detection architecture is fast due to some design related reasons. First, the entire image is located inside FPGA memory [16 18]. This allows access to several image pixel values in a single clock cycle which enables pipelining of the edge detection process. Second, parallelism is used for performing horizontal and vertical convolutions simultaneously. Moreover, the Sobel, Prewit or Robert modules can be run simultaneously for generating multiple output images.

The rest of the paper is organized as follows. Sobel, Prewit and Robert edge detection operations are presented in Section 3 The proposed FPGA-based edge detection architecture is presented in Section 4 and Section 5 calculates the speed-up of the process. Sec- 
tion 6 provides experimental results and finally Section 7 concludes the paper.

\section{RELATED WORKS}

As a new trend towards real-time image processing, some first order derivative edge detection methods have been implemented on

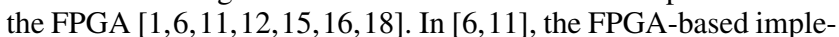
mentation of some first order derivative edge detectors like Sobel, Prewit and Robert is presented. They use Xilinx system generator to generate synthesizable HDL codes for FPGA-based edge detectors. Although Xilinx system generator makes implementation fast and simple, it doesnt lead to optimized HDL generation for area and speed. Moreover, Xilinx system generator is limited to using Xilinx board and MATLAB Simulink.

In [15], a parallel FPGA-based architecture is proposed to perform Sobel edge detection. In this method, a binary image is stored in a text file and it is loaded inside the FPGA memory. Then, two raster windows are used in parallel to perform both horizontal and vertical convolutions to compute gradient values. Although performing two convolutions in parallel leads to high speed, it still can be improved by introducing more levels of parallelism using pipeline architecture or working on different image regions simultaneously.

In [1], another implementation for Sobel edge detection is presented. This architecture uses dual port RAM and $3 \times 3$ pixel generator module to cache line of image data. Pixel generator includes 3 shift registers for each line that causes some delays to provide image pixel values for convolution module. This delay causes inefficient usage of pipeline since pipeline stalls due to fetching each pixel value.

In [18], the Sobel operation is controlled by Finite State Machine (FSM). In this method, there is a Sobel controller block that controls data flowing from SDRAM to FPGA RAM block. This also performs convolution process and updates the result into FPGA RAM blocks for next convolution. However, this method is not fast enough due to using RAM blocks.

The combination of hardware and software is presented in [12] that implements area efficient Sobel edge detection. Since fully parallel architecture cannot share hardware over multiple clock cycles, it uses serial architecture to minimize hardware resources. However, it is not optimized for speed since it uses serial architecture which is not well suited for fast architectures. [16] presents a fast Sobel edge detection hardware that performs convolution using addition and subtraction instead of multiplication. Their speed is accelerated by performing convolution in parallel way for finding horizontal and vertical edges. However, it can be improved using pipeline structure and other level of parallelism.

\section{FIRST ORDER DERIVATIVE EDGE DETECTION ALGORITHMS}

Image edges are one of the most fundamental features for object recognition in computer vision. Edge detection is a classical problem in image processing that identifies image locations that have sharp brightness changed or more formally, discontinuities [6].

First order derivative edge detection algorithms are more desired than second order derivative edge detection methods since they are more robust toward noise. They usually find edges by finding the minimum and/or maximum value in the first derivative of the image intensity values. It is often convenient to use gradient operators to obtain the first derivative of an image. There are various types of the gradient operators, such as Roberts operator [6], Prewit operator

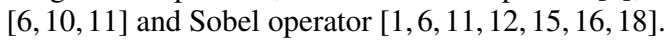

We have considered Sobel Prewit and Robert operators for our edge detection implementation in hardware. Sobel and Prewit use two $3 \times 3$ spatial masks and Robert uses two $2 \times 2$ spatial masks to find image edges. These masks are used for computing the gradient of the image intensity values in horizontal and vertical directions. The Sobel, Prewit and Robert masks, which are also called convolution kernels, are shown in Fig. 11(a)-(c).

By sliding the convolution kernels over the image, the gradient of the image intensity values along $\mathrm{x}$ and $\mathrm{y}$ directions is computed. Fig. 1 (d) shows intensity values of an image for a typical $3 \times 3$ sub-window. By applying convolution masks on the sub-window, horizontal $\left(G_{x}\right)$ and vertical edges $\left(G_{y}\right)$ are computed. Formulas for computing $G_{x}$ and $G_{y}$ using convolution masks for Sobel, Prewit and Robert operations are shown in 12 and 3 respectively.

$$
\begin{gathered}
G_{x}=(D 6+2 D 7+D 8)-(D 0+2 D 1+D 2) \\
G_{y}=(D 0+2 D 3+D 6)-(D 2+2 D 5+D 8) \\
G_{x}=(D 0+D 1+D 2)-(D 6+D 7+D 8) \\
G_{y}=(D 2+D 5+D 8)-(D 0+D 3+D 6) \\
G_{x}=(D 4)-(D 8) \\
G_{y}=(D 5)-(D 7)
\end{gathered}
$$

The magnitude of gradients for all three operations is obtained by 4

$$
|G|=\sqrt{G_{x}^{2}+G_{y}^{2}}
$$

However, it is not convenient to calculate magnitude of gradients in hardware by 4 since it cannot be computed in a single clock cycle as it is desired for pipeline structure. Alternatively, magnitude can be estimated by 5 Since it can get computed in one clock cycle, it is used in our edge detection hardware.

$$
|G|=\left|G_{x}\right|+\left|G_{y}\right|
$$

Although the magnitude implies the strength of the edge at pixel location $(x, y)$, it is not informative for the direction of the edge [15] [18]. The gradient direction is computed by 6

$$
\alpha(x, y)=\tan ^{-1}\left(\frac{G_{y}}{G_{x}}\right)
$$

The edge detection process for Sobel, Prewit and Robert operations are similar and the only difference is due to using different convolution masks.

\section{FPGA-BASED EDGE DETECTION ARCHITECTURE}

The FPGA-based architecture for edge detection is shown in Fig. 2. Three separated hardware modules are defined to perfrom Sobel, Prewit and Robert operations. Each hardware is enabled by a $3 \times 8$ decoder that has three inputs (A, B and C). Table 1 shows the relation between inputs and corresponding edge detection modules. For example, if A, B and C become 0, 0 and 1 respectively, Sobel edge detection hardware is enabled. Modules are organized in parallel that lead them to perform edge detection simultaneously. For example, if A, B and C become 1, all three edge detection modules 


\begin{tabular}{|c|c|c|c|c|c|}
\hline-1 & -2 & -1 & 1 & 0 & -1 \\
\hline 0 & 0 & 0 & 2 & 0 & -2 \\
\hline 1 & 2 & 1 & 1 & 0 & -1 \\
\hline
\end{tabular}

(a)

\begin{tabular}{|c|c|c|c|c|c|}
\hline 1 & 1 & 1 & -1 & 0 & 1 \\
\hline 0 & 0 & 0 & -1 & 0 & 1 \\
\hline-1 & -1 & -1 & -1 & 0 & 1 \\
\hline
\end{tabular}

(b)

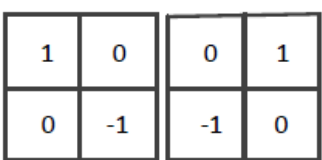

(c)

\begin{tabular}{|l|l|l|}
\hline D0 & D1 & D2 \\
\hline D3 & D4 & D5 \\
\hline D6 & D7 & D8 \\
\hline
\end{tabular}

(d)

Fig. 1: Convolution kernels for gradient operations: (a) Sobel (b) Prewit (c) Robert, d) Intensity values of typical $3 \times 3$ sub-window

Table 1. : Relation between inputs and enabling edge detection modules

\begin{tabular}{cccc}
\hline \hline \multicolumn{3}{c}{ Inputs } & Operation \\
A & B & C & Nothing \\
\hline 0 & 0 & 0 & Sobel \\
\hline 0 & 0 & 1 & Prewit \\
\hline 0 & 1 & 0 & Robert \\
\hline 0 & 1 & 1 & Sobel+Prewit \\
\hline 1 & 0 & 0 & Sobel +Robert \\
\hline 1 & 0 & 1 & Sobel +Prewit + Robert \\
\hline 1 & 1 & 0 & \\
\hline 1 & 1 & 1 & \\
\hline \hline
\end{tabular}

are enabled and three different operations are done at the same time in parallel way. As result, the run time of one operation is same as having two or even three different operations.

As it is shown in Fig. 2, each hardware module has 72 bits input from the FPGA memory which stores intensity values of the original image. This is due to fetching 9 intensity values (i.e. $9 \times 8$ bits) for each edge detection modules at every clock cycle.

\subsection{Pipeline Structure}

Each edge detection module uses pipeline to speed-up the edge detection process of an image located inside FPGA memory. Since the edge detection operation is performed by two convolution kernels in parallel, two different pipelines are used for each module. Since there is no dependency between pipeline stages, they are able to perform the edge detection process at each clock cycle without getting stalled. Fig. 3 shows 4 stages of the proposed pipeline structure.

(1) Fetch: 9 intensity values are read for two separated pipelines $\left(G_{x}\right.$ and $\left.G_{y}\right)$ of each module. Six intensity values (D0, D1, D2, D6, D7, D8) are used for computing horizontal gradient $\left(G_{x}\right)$ and six intensity values (D0, D3, D6, D2, D5, D8) are used for computing vertical gradient $\left(G_{y}\right)$ of the Sobel and Prewit modules. Four intensity values (D4, D8) and (D5, D7) are used to compute $\left(G_{x}\right)$ and $\left(G_{y}\right)$ of Robert module. So, the number of pipeline registers in Robert module is less than two other modules. Intensity values are read at one clock cycle and they are passed to the convolution stage by next clock cycle.

(2) Convolution: $G_{x}$ and $G_{y}$ are computed for each pixel located at the center of $3 \times 3$ sub-window (D4). As it was shown in 1 and 2 , four additions, two multiplications and one subtraction circuits are needed to compute $G_{x}$ or $G_{y}$ (Soble, Prewit

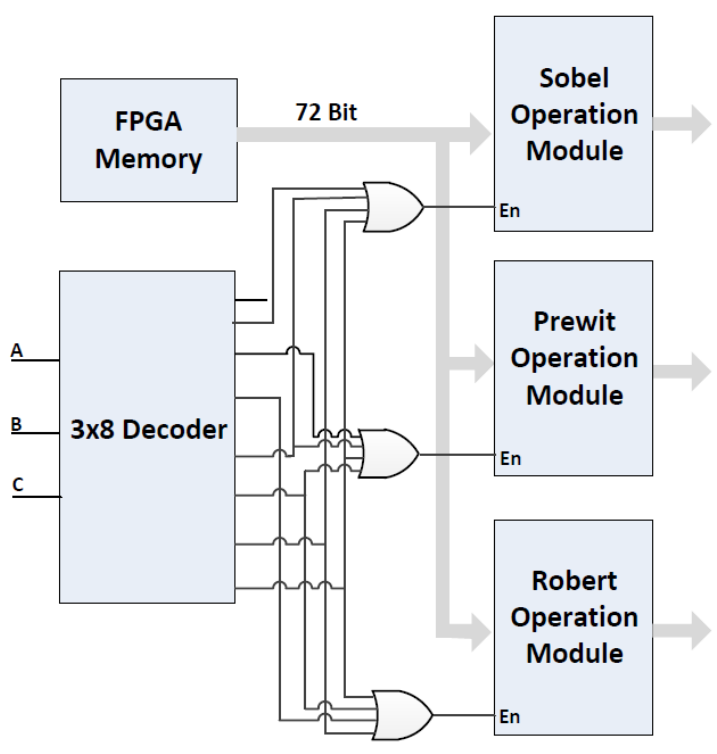

Fig. 2: FPGA-based edge detection architecture

modules). Based on 3 the Robert module only needs one subtraction circuit for either $G_{x}$ or $G_{y}$. Having separated combinational circuits allows us to perform convolutions in parallel for $\left(G_{x}\right)$ and $\left(G_{y}\right)$. As it is shown in Table 1 if more than one module is enabled, pipelines of each related modules are run simultaneously.

(3) Absolute Value of $G_{x}, G_{y}$ : Computed $G_{x}$ and $G_{y}$ values of previous stage, are checked against zero to determine they are positive or negative. If they are negative, they are multiplied by -1 to compute the absolute value in a single clock cycle.

(4) $|G|=\left|G_{x}\right|+\left|G_{y}\right|$ : The gradient magnitude is computed using 5 by adding the absolute magnitude values of $G_{x}$ and $G_{y}$ Although the true magnitude $G$ can be computed by 4 the full implementation needs conditional loop instructions that will need more than one clock cycle. It means that the instruction should be executed several times based on the condition. Since each pipeline has four stages, four clock cycles are needed to fill the pipeline. After each pipeline is filled, a final gradient result $(|G|)$ is generated at each clock cycle. This architecture is effective since there are no data or control hazards between pipeline stages and it could be run at full speed with no interruption [5]. 


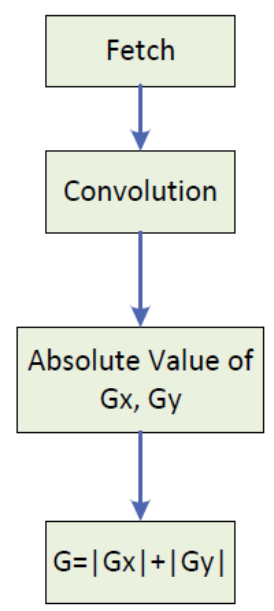

Fig. 3: Pipeline stages for each edge detection module

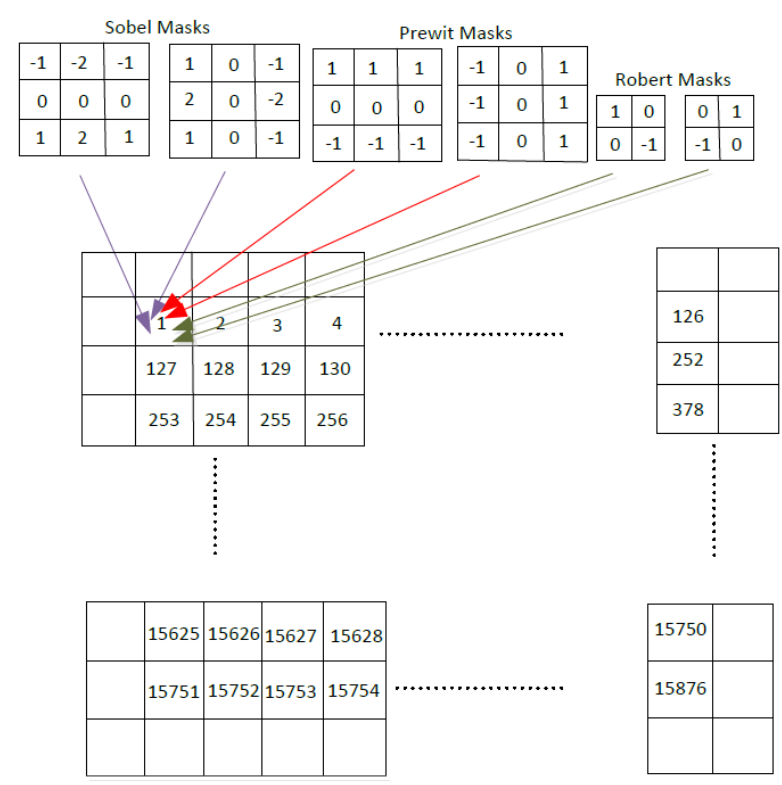

Fig. 4: One-way parallel method. Since intensity values on borders of an image are not considered for edge detection process, we have not assigned number to them.

\subsection{One-way Parallel Edge Detection Architecture}

one-way parallel method works based on performing edge detection at one region of an image. One region is the whole intensity values of an image excepting borders. In one-way parallel method, nine intensity values related to each pixel is fetched in row order. So, multiplication by convolution masks is done at the next clock cycle in parallel to compute $G_{x}$ and $G_{y}$.

Fig. 4 depicts the simple picture of the method for the image size of $128 \times 128$. Firstly, 9 intensity values regarding to pixel 1 are read to form the $3 \times 3$ sub-window. Fetching 9 intensity values continues in row order from pixel 2 to 15876 . A separated pipeline is used inside each module for each convolution kernel. Pipeline operations are explained in clock cycle order as below.

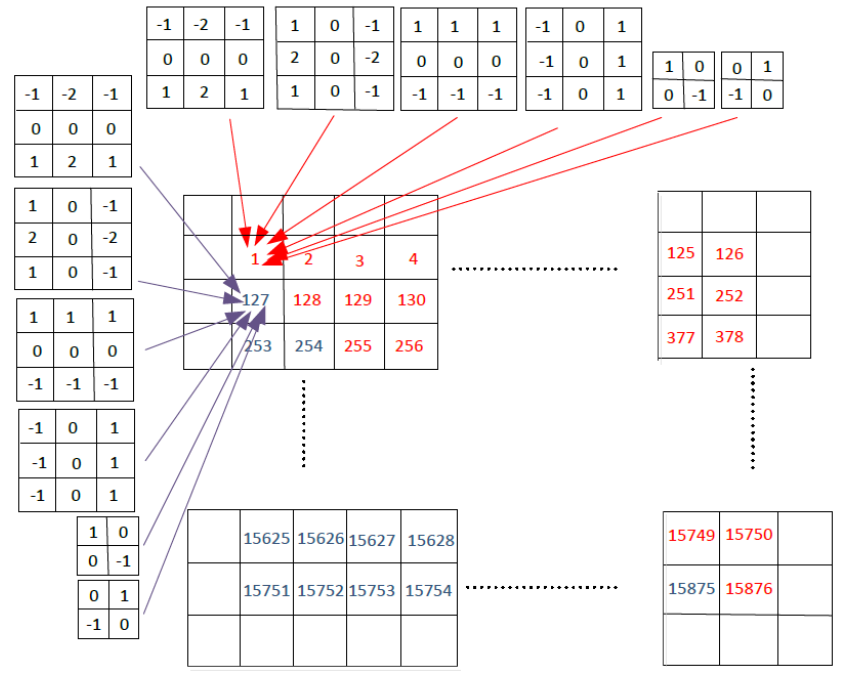

Fig. 5: Two-way parallel method, two separated regions are shown by two different colors

(1) Pipeline starts with pixel 1 and it fetches 9 related intensity values to form the $3 \times 3$ sub-window at first clock cycle.

(2) While 9 intensity values related to pixel 2 are fetched, 9 intensity values regarding to pixel 1 are already passed to convolution stage. It means that at the same clock cycle, pixel 2 is fetched and convolution for pixel 1 is computed. This convolution is made for both horizontal and vertical convolution masks at the same clock cycle.

(3) 9 intensity values related to pixel 3 are fetched, convolution for pixel 2 is performed and absolute values of $G_{x}$ and $G_{y}$ are computed for pixel 1 .

(4) 9 intensity values related to pixel 4 are fetched, convolution is performed (pixel 3), absolute values of $G_{x}$ and $G_{y}$ are computed (pixel 2) and the final magnitude for absolute values $|G|$ is computed (pixel 1). Pipelines get filled after four clock cycles and final magnitude value $(G)$ is calculated for each pixel by one clock cycle.

\subsection{Two-way Parallel Edge Detection Architecture}

The two- way parallel edge detection architecture extends the oneway process by defining two separated regions in the original image. Convolution is perfromed for each region separately by introducing additional convolution kernels and pipelines.

Fig. 5 depicts the operation of the two-way parallel method. Two groups of image intensity values, which split the image along the diagonal, are distinguished by different colors. Two convolution masks and two separated pipelines are used for each edge detection module regarding to each group. For example, fetching of 9 intensity values for pixel 1 and 127 is perfromed simultaneously. Pipelines are run in full speed since there is no data dependency between two pipelines. This effectively doubles the speed in comparison with one-way method.

The idea of two-way parallel method could also be extended towards $\mathrm{m}$-way parallel method by defining $\mathrm{m}$ regions on the original image. This extension is effective for increasing the speed-up but it suffers from an extra overhead related with each additional pipeline structure. The parallelism is limited by the FPGA hard- 
ware. We later show that going towards m-way of parallelism is effective when the image size increased.

\section{SPEED-UP CALCULATION}

Any hardware that does not use a pipeline must complete each task to finish an operation. If there are $n$ tasks to be executed, and each task needs $t$ time then total runtime is obtained by 7

$$
T_{\text {total }}=n t
$$

If each task needs $k$ number of clocks to be completed, 8 is obtained [5]13.

$$
T_{\text {total }}=n k \times c l k
$$

where $c l k$ represents the clock cycle time. The total runtime of the same task using a pipeline is obtained by 9

$$
T_{\text {total-pipeline }}=(k+n-1) \times c l k
$$

Speed-up of a hardware benefits pipeline over the equivalent hardware is defined by the ratio in 10

$$
\text { speed }-u p=\frac{n k \times c l k}{(k+n-1) \times c l k}=\frac{n k}{k+n-1}
$$

As the number of tasks increased, $\mathrm{n}$ becomes too larger than $\mathrm{k}$ and the speed-up under this condition is simplified by 11 .

$$
\text { speed }-u p=\frac{n k}{k}=k
$$

This means that even when there are many instructions, speedup gets close to number of the pipeline stages. Therefore, more pipeline stages are desired for greater speed-up.

9 can be easily used to estimate the run time of the edge detection process for one-way parallel method,. For example, we just need to plug in 15870 as number of instructions $(n), 4$ as number of each pipeline stages $k$ and $\frac{1}{\text { Clock Frequency }}$ as $c l k$ for runtime estimation in Table. 3 .

In order to reach to m-way parallelism, 9 should get changed since the longer path determines the total run time of the process. As result, 12 is obtained to calculate the total runtime when there is different instructions regarding to each path.

$$
T_{\text {total-pipeline }}=\left(\max \left(n_{1}, n_{2}, \ldots, n_{m}\right)-1+k\right) \times c l k
$$

Where $m$ is number of different parallel regions that each operates on its own pipeline and $k$ is the number of pipeline stages. These variables are set for runtime estimation of two-way parallelism by plugging 2 into $m, 4$ into $k$ and $\frac{1}{\text { Clock Frequency }}$ into $\mathrm{clk}$. Note, $n_{1}=\frac{126 \times 127}{2}, n_{2}=\frac{125 \times 126}{2}$ and $\max \left(n_{1}, n_{2}\right)$ is 8001 .

\section{EXPERIMENTAL RESULTS}

This section includes three parts. In first part, the implementation results of the one-way and two-way parallel architectures for a given $128 \times 128$ image size is presented. In second part, the runtime of proposed methods for different image sizes are evaluated and the speed-up is estimated. Finally, an extension to higher level of parallelism is proposed when the image size increased in order to guarantee high speed-up. (a)
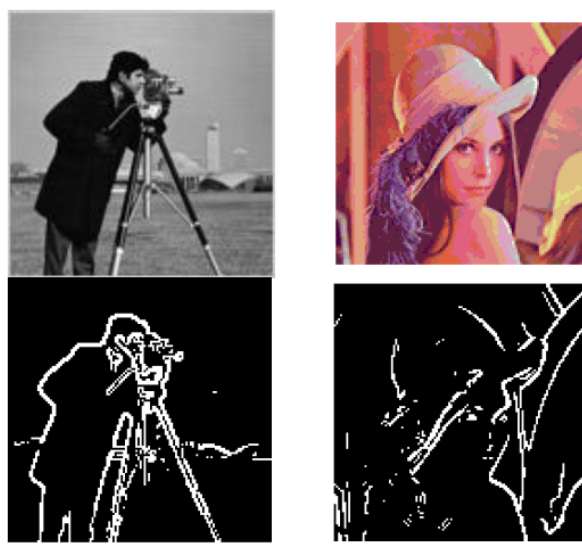

(c)
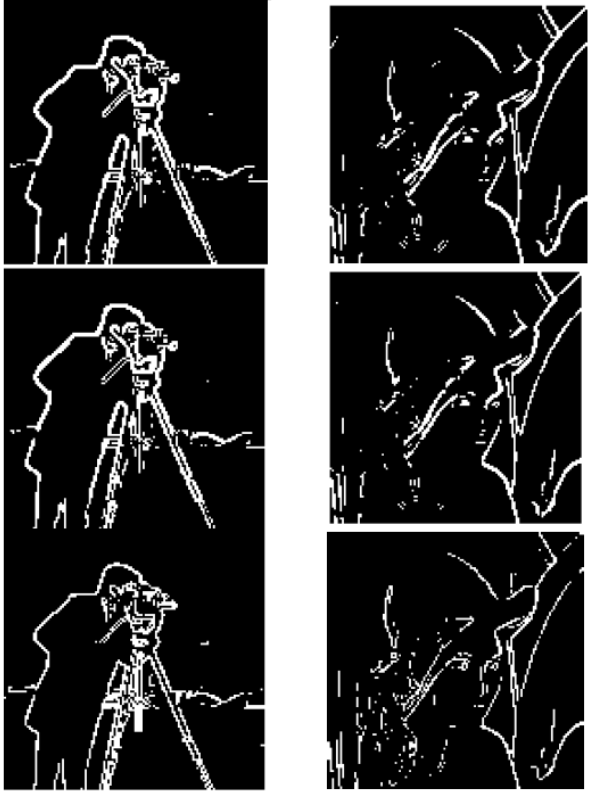

(d)

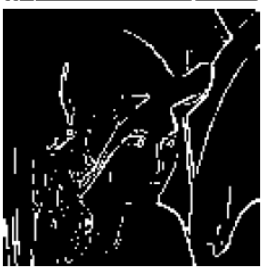

Fig. 6: Edge detection of Lenna \& Camera man images, (a) Original images (b) Sobel edge detection (c) Prewit edge detection (d) Robert edge detection

\subsection{Implementation}

One-way and two-way parallel architectures for a given $128 \times 128$ image was implemented by Verilog HDL and it was simulated using ModelSim 10.1d from Mentor Graphics Corporation. All images were first read and pre-processed using MATLAB R2010a to resize the image to $128 \times 128$ pixels, convert it to grayscale image, and write the pixel intensity values into a text file in hexadecimal format. The text file is created to use in the hardware simulator; it is loaded into the FPGA memory and read by the edge detection hardware.

Filtered pixels are written into an output text file at each clock cycle after the pipeline initialization. Another MATLAB script rearranges the filtered image pixels into a $128 \times 128$ final image after thresholding. As noted in Table. 11, two or even three filtered output pixels can be generated in a single clock cycle.

Fig. 6 shows the original and final images after performing edge detection by Robert, Sobel and Prewit modules. Robert shows more missed edges and both Sobel and Prewit detect thicker edges as it is expected.

We synthesized both edge detection architectures with Quartus II 9.1 web Edition for the Cyclone IV GX FPGA. Table. 2 shows the hardware resources that were utilized for EP4CGX150DF31C8 FPGA.

The one-way architecture consumes $18 \%$ of the FPGA memory and it gets double for two-way architecture. The two-way architecture requires twice the number of the registers due to convolution ker- 
Table 2. : Device utilization summery for one \& two-way parallel architectures

\begin{tabular}{ccccc}
\hline \hline & & Pins & Logic Elements & Memory Bits \\
\hline & Available & 508 & 149760 & 6635520 \\
\hline \multirow{2}{*}{ One-way } & Used & 78 & 925 & 1179648 \\
& Utilization & $15 \%$ & $1 \%$ & $18 \%$ \\
\hline \multirow{2}{*}{ Two-way } & Used & 143 & 1588 & 2359296 \\
& Utilization & $28 \%$ & $1 \%$ & $36 \%$ \\
\hline \hline
\end{tabular}

Table 3. : Runtime results for first \& second FPGA-based architectures

\begin{tabular}{ccccc}
\hline \multirow{2}{*}{ Method } & \multicolumn{2}{c}{ MATLAB } & \multicolumn{2}{c}{ Architecture } \\
& Lenna & Camera man & First & Second \\
\hline Sobel & 0.12581 & 0.12256 & 0.00031 & 0.00016 \\
\hline Prewit & 0.12348 & 0.12303 & 0.00031 & 0.00016 \\
\hline Robert & 0.11521 & 0.11682 & 0.00031 & 0.00016 \\
\hline Sobel+Prewit & 0.15552 & 0.14476 & 0.00031 & 0.00016 \\
\hline Prewit+Robert & 0.15871 & 0.15128 & 0.00031 & 0.00016 \\
\hline Sobel+Robert & 0.15435 & 0.14839 & 0.00031 & 0.00016 \\
\hline Sobel +Prewit+Robert & 0.16342 & 0.15330 & 0.00031 & 0.00016 \\
\hline
\end{tabular}

nels of two pipelines. Since some combinational circuits are shared between pipelines, total logic elements are not exactly get doubled. Consumed logic elements of second architecture increased by rate of 1.71 but it still consumes $1 \%$ due to large number of available logic elements.

Table. 3 presents the runtime of the edge detection process on the FPGA with $50 \mathrm{MHZ}$ clock frequency. The runtime of Soble, Prewit and Robert functions are measured in MATLAB R2010a on a machine with 6 Gigabyte RAM and i7 processor. The processor includes 8 cores with a $2.00 \mathrm{GHZ}$ clock frequency. The average of 10 runtimes are accounted for MATLAB and positive edge triggered counters are used to estimate runtime on FPGA.

Table. 3 shows more speed-up for multiple edge detection filters. Although some parallelism is performed using the multiple processor cores for MATLAB, it cannot fully execute edge detection processes in parallel because of its sequential nature. More edge computation increases runtime in software but FPGA has constant run-time for all combinations due to usage of parallelism between edge detection modules.

Runtime of edge detection process for two-way parallel architecture is half of the one-way architecture. This is a direct result of the transition from one-way parallelism to two-way parallelism. Although experimental results show large speed-up, the hardware speed-up depends on the image size. The speed-up calculation for different image sizes and the architecture extension toward m-way parallelism is presented in following sub-sections.

\subsection{Speed-up analysis}

Formulas 9 and 12 are used to estimate the runtime of the proposed methods on FPGA and MATLAB run edge detection functions for different image sizes. Fig. 7 shows the run-time of oneway and two-way parallel methods versus the software-based approach when the image size scales by factor of 4 . As it is was shown

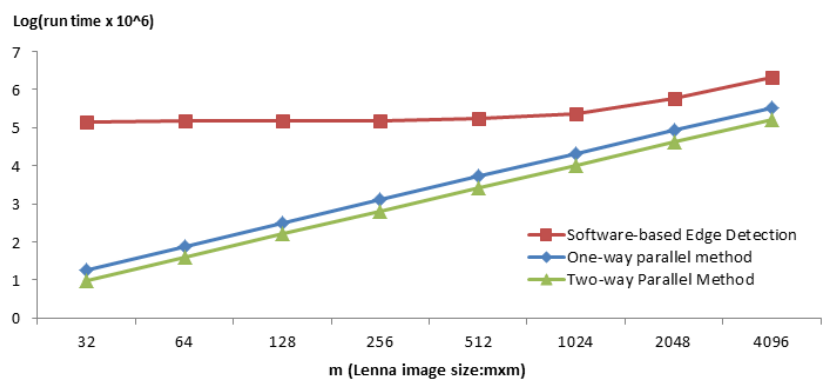

Fig. 7: Run time of software-based edge detection versus one-way and twoway parallel method for different image sizes

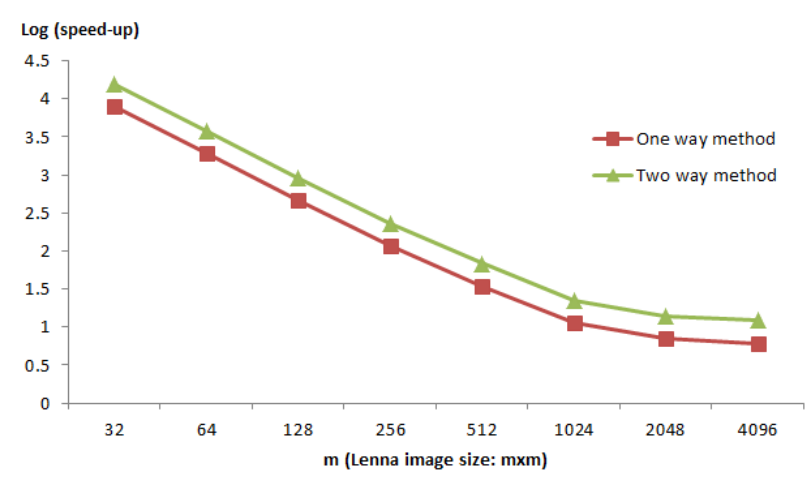

Fig. 8: Speed-up for different image sizes of Lenna Image

in 9 runtime for one-way and two-way methods is linearly related with the image size. If the image size increased by factor of 4 , the runtime increased quadratically.

MATLAB R 2010a was used to run each edge detection method for different image sizes. Lenna image was up-sampled by factor of four and the average of worst and best 10 runtimes was measured. Best case is usually achieved when Robert edge detection is only used due to its smaller kernel mask. On the other hand worst case happens when all three edge detections are needed to get run for the given image. The average plot is shown for software-based edge detection in Fig. 7

When the image size is less than $1024 \times 1024$, runtime of the edge detection process does not change significantly. It means that the run time of the software-based approach doesnt necessarily follow formula 8 and it doesnt linearly increased due to instruction and data level parallelism on multi-cores [5]. However, when image size is larger than 1024, the run time increased as similar as the proposed parallel methods.

Fig. 8 shows the speed-up results. Speed-up is near to 1000 (logarithm of speed-up is near to 3 ) for $128 \times 128$ image in two-way parallel method and it is near to 400 for $256 \times 256$ image. However, it goes below 30 when the image size becomes higher than 1024. These effects are shown as plunge in speed-up results in Fig. 8 . Although ordinary FPGAs might not have enough memory bits to keep the large size images, we should find a way to deal with this issue for those with enough memory bits. In next section, the higher level of parallelism is proposed for solving this problem. The $\mathrm{m}$-way parallel approach guarantees a non-decreasing speed-up of edge detection on large image sizes. 

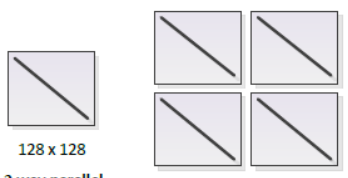

2 way paralle

$256 \times 256$

8 way parallel

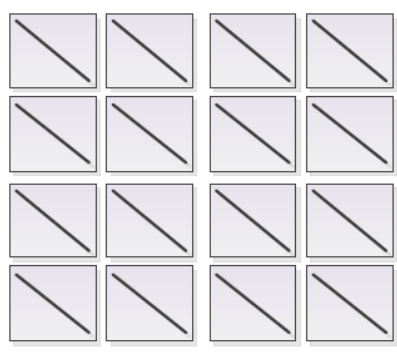

$512 \times 512$

32 way paralle
Method

Fig. 9: Extending the parallel approach when the image size gets increased

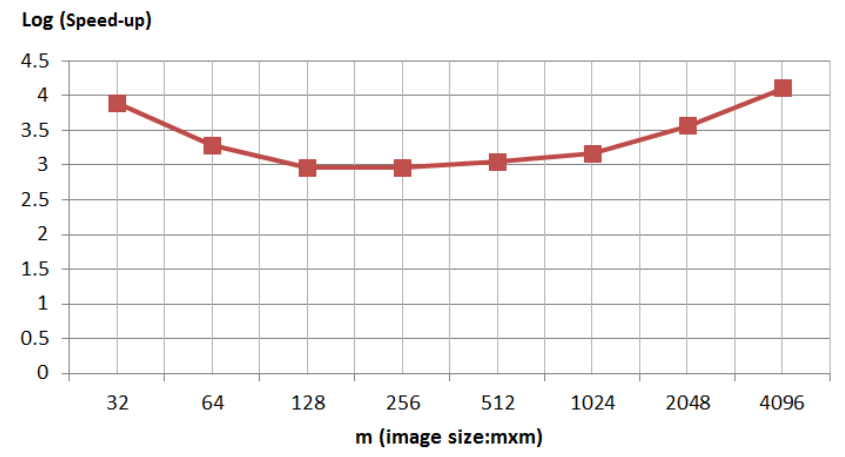

Fig. 10: Final Speed-up using increasing m-way method

\subsection{Extension toward m-way parallelism}

The extension from 1-way to 2-way and m-ways was shown in formula 12 This parallelism extension is beneficial for large image sizes. Transition from one-way to two-way parallel method was previously shown by considering two triangle areas over the image intensity values. The region that covers more intensity values has a longer path and it determines the total runtime. As simple solution, the parallelism can increased when the image size increased.

Fig. 9 shows the transition from 2-way to 8-way, when the image size increased from $128 \times 128$ to $256 \times 256$. The same process can get repetated from 8 way to 32 way when image scales to $512 \times 512$. Larger image sizes can still be broken into smaller sub-images by utilizing simple two-way parallel blocks. The run time of the full image is still same as the $128 \times 128$ image. It has runtime of twoway parallel method and larger upper triangle still determines the runtime.

Fig. 10 shows the speed-up for increasing m-way. When the image size is between $128 \times 128$ and $1024 \times 1024$, speed-up is around 1000 and it does not drop for greater image sizes. It actually increased for larger images since software approach can no longer efficiently utilize the multiple cores.

\section{CONCLUSION}

A fast FPGA-based architecture for performing first-order derivative edge detection is presented using a general approach for applying different levels of parallelism. Two architectures are proposed that benefit pipeline structure along with different levels of parallelism. Different levels of parallelism are introduced over convolution masks, hardware edge detection modules and image inten- sity values. The parallel hardware architecture achieved significant speed-up over software especially when multiple edge detectors are required. Finally, an extension of the proposed method towards mlevel parallelism is presented to guarantees non-decreasing speedup for large image sizes.

\section{REFERENCES}

[1] G. Anusha, T. J. Prasad, and D. Narayana. Implementation of sobel edge detection on fpga. Proceeding of the International Journal of Computer Trends and Technology, 3(3):471-475, 2012.

[2] D. G. Bariamis, D. K. Iakovidis, D. E. Maroulis, and S. A. Karkanis. An fpga-based architecture for real time image feature extraction. In Proceedings of the 17th International Conference on Pattern Recognition, pages 801-814, Cambridge, UK, 2004.

[3] M. P. S. Chikkali and K. Prabhushetty. Fpga based image edge detection and segmentation. Proceeding of the International Journal of Advanced Engineering Sciences and Technologies, 9(2):187-192, 2011

[4] B. A. Draper, J. R. Beveridge, A. P. W. Bhm, C. Ross, and M. Chawathe. Accelerated image processing on fpgas. IEEE Transactions on Image Processing, 12(12):1543-1551, 2003.

[5] J. L. Hannesy and D. A. Patterson. Computer Architecture, A Quantitative Approach. Morgan Kaufmann, 5 edition, 2011.

[6] R. Harinarayan, R. Pannerselvam, M. M. Ali, and D. K. Tripathi. Feature extraction of digital aerial images by fpga based implementation of edge detection algorithms. In Proceeding of the International Conference on Emerging Trends in Electrical and Computer Technology, pages 631-635, Tamil Nadu, India, 2011.

[7] W. Jincheng, Sun Jingrui, and Liu Wenying. Design and implementation of video image edge detection system based on fpga. In Proceedings of the 3rd International Congress on Image and Signal Processing, pages 472-476, Yantai, China, 2010.

[8] C. T. Johnston, K. T. Gribbon, and D. G. Bailey. Implementing image processing algorithms on fpgas. In Proceeding of the Eleventh Electronics New Zealand Conference, pages 118-123, Palmerston North, New Zealand, 2004.

[9] D. Llamocca, C. Carranza, and M. Pattichis. Separable fir filtering in fpga and gpu implementations: Energy, performance, and accuracy considerations. In Proceedings of the International Conference on Field Programmable Logic and Applications, pages 363-368, Chania, 2011.

[10] R. Maini and J. S. Sohal. Performance evaluation of prewit edge detector for noisy images. Proceeding of the International Journal on Graphics, Vision and Image Processing, 6(3):90-95, 2006. December.

[11] S. K. C. J. Majumdar. A novel architecture for real time implementation of edge detectors on fpga. Proceeding of the International Journal of Computer Science Issues, 8(1):193-202, 2011.

[12] R. Mehra and S. Verma. Area efficient fpga implementation of sobel edge detector for image processing application. Proceeding of the International Journal of Computer Applications, 56(16), 2012.

[13] D. A. Patterson and J. L. Hannesy. Computer Organization and Design, The Hardware/Software Interface. Morgan Kaufmann, 5 edition, September 2013. 
[14] J. M. Ramirez, E. M. Flores, j. Martinez-Carballido, R. Enriquez, V. Alarcon-Aquino, and D. Baez-Lopez. An fpgabased architecture for linear and morphological image filtering. In Proceeding of the 20th International Conference on Electronics, Communications and Computer, pages 90-95, Cholula, Mexico, 2010.

[15] V. Sanduja and R. Patial. Sobel edge detection using parallel architecture based on fpga. Proceeding of the International Journal of Applied Information Systems, 3(14), 2012.

[16] H. Santanu, D. Bhattacharjee, M. Nasipuri, and D. K. Basu. A fast fpga based architecture for sobel edge detection. In Proceeding of the 16th International Conference on Progress in VLSI Design and Test, pages 300-306, Shibpur, India, 2012. Springer Berlin Heidelberg.

[17] R. Szeliski. Computer Vision: Algorithms and Applications. Morgan Kaufmann, 2010.

[18] I. Yasri, N. H. Hamid, and V. V. Yap. Performance analysis of fpga based sobel edge detection operator. In Proceeding of the International Conference on Electronic Design, pages 1-4, Penang, Malaysia, 2008. 